\title{
A first-principles analysis of energy transport by oscillatory waves
}

\author{
O. Maj \\ Max Planck Institute for Plasma Physics, EURATOM Association, Garching, Germany, and \\ Max Planck Institute for Solar System Research, Katlenburg-Lindau, Germany
}

\begin{abstract}
The asymptotic solution of the initial value problem for Maxwell's equations in the highfrequency limit is applied to the derivation and discussion of the main radiative energy transport models. Particularly, the form of the radiative transfer equation relevant to non-stationary plasmas is addressed.
\end{abstract}

\section{INTRODUCTION AND MATHEMATICAL SETTING}

In addition to its relevance as a problem in mathematical physics [1], the precise derivation of radiative energy transport models is useful whenever phenomenological derivations yield unclear results. This is the case when plasma parameters depend on time, and two different forms of the radiative transfer equation exist in literature, one reported by Bekefi [2], cf. also [3], and the other one reported by Stix [4], cf. also [5].

We address the derivation and the rigorous justification of transport models for the wave energy density carried by a random spectrum of oscillatory waves in the high frequency limit. The main tool of the analysis is the semiclassical calculus of pseudodifferential operators and oscillatory integrals on the lines of a previous work [6], with the semiclassical parameter $\varepsilon=\lambda / L \rightarrow 0, \lambda$ being the typical wave-length of the oscillatory waves and $L$ being the typical scale length of the parameters of the plasma (let us recall that, roughly speaking, a pseudodifferential operator is an integral operator with a kernel that can be written as the Fourier transform of a tensor, called symbol of the operator, such as the plasma dielectric tensor). The presentation is rather formal and some mathematical results will be given without proof.

The equation for the wave electric field in a non-uniform dispersive medium can be written in the abstract form

$$
P^{\varepsilon} u^{\varepsilon}(t, x)=f^{\mathcal{E}}(t, x),
$$

where $P^{\varepsilon}$ is a matrix of pseudodifferential operators acting on the normalized wave electric field $u^{\varepsilon}$, and $f^{\varepsilon}$ represents embedded sources accounting for various emission processes [5]; here, $t$ and $x=\left(x_{1}, x_{2}, x_{3}\right)$ denote time and spatial position normalized to the typical scales of time- and space-variation of the plasma. We recall that the Weyl symbol map $[1,7]$ relates the operator $P^{\varepsilon}$ to its Weyl symbol $p^{\varepsilon}$ which reads,

$$
p^{\varepsilon}(t, x, \tau, \xi) \sim \sum_{j \geq 0} \varepsilon^{j} p_{j}(t, x, \tau, \xi),
$$


where $\tau, \xi$ are dual coordinates to $t, x$; the space spanned by $(t, x, \tau, \xi)$ is the extended wave phase space. The leading order term $p_{0}(t, x, \tau, \xi)$ is just the local dispersion tensor which, in particular, depends on the Hermitian part of the local plasma dielectric tensor. As customary for WKB analysis, we make the following assumptions.

H1. The waves are weakly absorbed, i.e., the dispersion tensor $p_{0}$ is Hermitian.

H2. No mode conversion occurs, i.e., the eigenvalues $\lambda_{i}$ of $p_{0}$ are distinct, in the sense that $\left|\lambda_{i}-\lambda_{j}\right| \geq C>0$ uniformly for some strictly-positive constant $C$.

H3. Weakly driven medium, i.e., $f^{\varepsilon}=\varepsilon f_{1}^{\varepsilon}$.

Assumption H1, in particular, does not imply that absorption is totally disregarded as $p_{1}$ accounts for the $O(\varepsilon)$ anti-Hermitian part of the plasma local dielectric tensor.

According to Littlejohn and Flynn [7], under the foregoing hypotheses normal modes of the plasma can be readily singled out, and (1) can be reduced to a set of independent scalar equations for the field carried by each mode. Specifically, one looks for an elliptic semiclassical pseudodifferential operator $R^{\varepsilon}$ such

$$
P^{\varepsilon} R^{\varepsilon} \sim R^{\varepsilon} \tilde{P}^{\varepsilon}
$$

where $\tilde{P}^{\varepsilon}$ is a diagonal matrix of pseudodifferential operators (to be determined). If such an operator exists, then the components of the transformed field $\left(R^{\varepsilon}\right)^{-1} u^{\varepsilon}$ are decoupled and can be referred to as eigenmodes. This is done iteratively by making use of the Weyl symbol map which turns the composition of operators into the semiclassical MoyalWeyl star product $\left(*_{\varepsilon}\right)$ of their symbols; explicitly, $a *_{\varepsilon} b=a b+\frac{\varepsilon}{2 i}\{a, b\}+O\left(\varepsilon^{2}\right)$ for any two functions $a, b$ with Poisson brackets $\{a, b\}=\partial_{\zeta} a \partial_{z} b-\partial_{z} a \partial_{\zeta} b$ in the extended phase space $(z, \zeta)=(t, x, \tau, \xi)$ and straightforward generalizations for matrix-valued symbols of the form (2). For (3), one has $p^{\varepsilon} *_{\varepsilon} r^{\varepsilon} \sim r^{\varepsilon} *_{\varepsilon} \tilde{p}^{\varepsilon}$. The lowest order is thus given by $p_{0} r_{0}=r_{0} \tilde{p}_{0}$ : since $p_{0}$ is Hermitian $(\mathrm{H} 1)$, it can be diagonalized by a unitary transformation which means $\tilde{p}_{0}=\left(\lambda_{i} \delta_{i j}\right)$ where $\lambda_{i}$ are the eigenvalues of $p_{0}$ and $r_{0}$ is the unitary matrix obtained in terms of the corresponding eigenvectors (the polarization of plasma eigenmodes $)$. At the next order one has $p_{0} r_{1}-r_{1} \tilde{p}_{0}=r_{0} \tilde{p}_{1}-p_{1} r_{0}+\frac{1}{2 i}\left(\left\{r_{0}, \tilde{p}_{0}\right\}-\right.$ $\left.\left\{p_{0}, r_{0}\right\}\right)$; upon writing $r_{1}=r_{0} b_{1}$, multiplying by $r_{0}^{*}=r_{0}^{-1}$ and computing the commutator $\left[\tilde{p}_{0}, b_{1}\right]$ one finds $\left(\lambda_{i}-\lambda_{j}\right) b_{1, i j}=\tilde{p}_{1, i j}-\left(r_{0}^{*} p_{1} r_{0}\right)_{i j}+\frac{1}{2 i}\left[r_{0}^{*}\left(\left\{r_{0}, \tilde{p}_{0}\right\}-\left\{p_{0}, r_{0}\right\}\right)\right]_{i j}$. Since $\tilde{p}_{1}$ must be diagonal, we can determine the off-diagonal terms of $b_{1}$ from the off-diagonal terms of the equation; with this aim, assumption $\mathrm{H} 2$ is crucial. Then, the diagonal terms determine uniquely $\tilde{p}_{1}$. Iteration gives the symbol $r^{\varepsilon}$ to the required accuracy in $\varepsilon$. As a consequence, the $l$-th eigenmode satisfies

$$
\tilde{P}_{l l}^{\varepsilon} v_{l}^{\varepsilon} \sim g_{l}^{\varepsilon}=\varepsilon g_{1, l}^{\varepsilon},
$$

where $v^{\varepsilon}=\left(R^{\varepsilon}\right)^{-1} u^{\varepsilon}, g^{\varepsilon}=\left(R^{\varepsilon}\right)^{-1} f^{\varepsilon}$, and $\tilde{P}_{l l}^{\varepsilon}$ is the $(l, l)$ entry of the matrix $\tilde{P}^{\varepsilon}$. The solution of such an equation is written in terms of oscillatory integrals, namely,

$$
v_{l}^{\varepsilon}(t, x)=(2 \pi \varepsilon)^{-3} \int e^{\frac{i}{\varepsilon} \phi_{l}(t, x, \alpha)}\left[a_{l}(t, x, \alpha) \hat{v}_{0, l}^{\varepsilon}(\alpha)+b_{l}^{\varepsilon}(t, x, \alpha)+O(\varepsilon)\right] d \alpha,
$$

where the auxiliary variable $\alpha$ is the normalized wave-vector at the initial time $t=0$ and $\hat{v}_{0, l}^{\varepsilon}(\alpha)$ are the initial spectra of each mode; both $\hat{v}_{0, l}^{\varepsilon}$ and $b_{l}^{\varepsilon}$ are $O(1)$ for $\varepsilon \rightarrow 0$. The 
phase $\phi_{l}$ is a solution of the relevant eikonal equation $\lambda_{l}\left(t, x, d \phi_{l}\right)=0$, which takes the form of a Hamilton-Jacobi equation $\partial_{t} \phi_{l}+H_{l}\left(t, x, d_{x} \phi_{l}\right)=0$ for $\lambda_{l} \propto \tau+H_{l}(t, x, \xi)$, with initial condition $\phi_{l}(0, x, \alpha)=x \cdot \alpha$. The amplitudes $a_{l}(t, x, \alpha)$ and $b_{l}^{\varepsilon}(t, x, \alpha)$ represent the evolution of the initial spectra and the field driven by the sources, respectively. The construction of the non-driven field follows the lines of the standard semiclassical calculus: $a_{l}$ satisfies the standard geometrical optics transport equation,

$$
V_{l} \cdot \partial a_{l}+\operatorname{div}\left(V_{l}\right) a_{l} / 2+i \tilde{p}_{1, l l} a_{l}=0
$$

with $V_{l}=d_{(\tau, \xi)} \lambda_{l}\left(t, x, d \phi_{l}\right)$, and with initial condition $a_{l}(0, x, \alpha)=1$. On the other hand, the treatment of the driven field is novel and constitutes a main result of this work. The substitution of (5) into (4) shows that $b_{l}^{\varepsilon}$ must be a solution of the inhomogeneous form of (6) with the source term $G_{1, l}^{\varepsilon}(t, x, \alpha)$ satisfying the condition

$$
g_{1, l}^{\varepsilon}=(2 \pi \varepsilon)^{-3} \int e^{\frac{i}{\varepsilon} \phi_{l}(t, x, \alpha)} G_{1, l}^{\varepsilon}(t, x, \alpha) d \alpha .
$$

Equation (7) is just the representation of the source term on the basis of the WKB wavelets $e^{\frac{i}{\varepsilon} \phi_{l}(t, x, \alpha)}$ labelled by $\alpha$, [6]. Let us also note that condition H3 is unavoidable as $\tilde{P}_{l l} v_{l}^{\varepsilon}$ is at least $O(\varepsilon)$. One finds $G_{1, l}^{\varepsilon}(t, x, \alpha)=e^{-\frac{i}{\varepsilon} \phi_{l}(t, x, \alpha)} \hat{g}_{1, l}^{\varepsilon}(t, x, \alpha)$ where $\hat{g}_{1, l}^{\varepsilon}=$ $\int e^{-\frac{i}{\varepsilon}\left(x-x^{\prime}\right) \cdot \alpha} g_{1, l}^{\varepsilon}\left(t, x^{\prime}\right) d x^{\prime}$ is the local Fourier transform of the source.

\section{RADIATIVE ENERGY TRANSPORT MODELS}

For physics applications, let $t, x$ be the non-normalized time and position (with some abuse of notations), and $\omega, k$ the frequency and wave-vector; the parameter $\alpha$ is kept normalized. The initial electric field spectra $\hat{v}_{0, l}^{\varepsilon}(\alpha)$, in many scenarios, are generated by random processes in the plasma and, therefore, they are regarded as random fields. Analogously let the local spectrum of the source be random as well, with the following assumptions on the statistical correlations: $\left\langle\hat{v}_{0, l}^{\varepsilon}(\alpha) \hat{v}_{0, l^{\prime}}^{\varepsilon}\left(\alpha^{\prime}\right)^{*}\right\rangle=\left|\mathscr{E}_{l}(\alpha)\right|^{2} \delta_{l l^{\prime}}(2 \pi \varepsilon)^{3} \delta(\alpha-$ $\left.\alpha^{\prime}\right),\left\langle\hat{v}_{0, l}^{\varepsilon}(\alpha) \hat{g}_{1, l^{\prime}}^{\varepsilon}\left(t, x, \alpha^{\prime}\right)^{*}\right\rangle=0$, and $\left\langle\hat{g}_{1, l}^{\varepsilon}(t, x, \alpha) \hat{g}_{1, l^{\prime}}^{\varepsilon}\left(t^{\prime}, x^{\prime}, \alpha^{\prime}\right)^{*}\right\rangle=\mathscr{G}_{l}\left(t, x, x^{\prime}, \alpha\right) \delta_{l l^{\prime}} \delta(t-$ $\left.t^{\prime}\right)(2 \pi \varepsilon)^{3} \delta\left(\alpha-\alpha^{\prime}\right)$, where $\mathscr{E}_{l}$ and $\mathscr{G}_{l}$ are the averaged spectral density of electric field and the spatial correlation of of the local spectrum of the sources, respectively. The foregoing assumptions, although physically reasonable, are not optimal and could be improved. By straightforward calculus, one can get the transport equation for the averaged wave action density $J_{l}(t, x, \alpha)$ in the $(x, \alpha)$ phase space directly from the geometric optics amplitude transport equation (6) with and without source. The result is

$$
\partial_{t} J_{l}+\operatorname{div}\left(v_{\mathrm{g}, l} J_{l}\right)=-2 \gamma_{l} J_{l}+p_{l},
$$

where $v_{\mathrm{g}, l}(t, x, \alpha)$ is the group velocity of the $l$-th mode, $\gamma_{l}$ is the absorption coefficient, and $p_{l}$ the emitted action density per unit volume in the phase space $(x, \alpha)$. It is worth noting that in a non-stationary plasma the wave action density $J_{l}=W_{l} / \omega_{l}$, rather than the wave energy density $W_{l}$, is the adiabatic invariant, i.e., it is conserved where $\gamma_{l}=0$ and $p_{l}=0$; here, $\omega_{l} \propto H_{l}$ is the eigenfrequency of the mode. 
In view of the specific initial conditions satisfied by the phases $\phi_{l}$, near $t=0$ the relationship $\alpha \rightarrow k=d_{x} \phi_{l}(t, x, \alpha)$ is one to one. When such a property persists for all time $t$, we can transform from the non-canonical phase space coordinates $(x, \alpha)$ to the canonical coordinates $(x, k)$. The crucial point for this transformation is the Jacobian $U_{l}(t, x, \alpha)=\operatorname{det}\left(\partial^{2} \phi_{l}(t, x, \alpha) / \partial x \partial \alpha\right)$ which satisfies $\partial_{t} U_{l}+v_{\mathrm{g}, l} \cdot \partial_{x} U_{l}+\operatorname{div}\left(v_{\mathrm{g}, l}\right) U_{l}=0$. By changing variable the wave action density $\tilde{J}_{l}(t, x, k)$ in canonical coordinates $(x, k)$ satisfies $J_{l}(t, x, \alpha)=U_{l}(t, x, \alpha) \tilde{J}_{l}\left(t, x, d_{x} \phi_{l}(t, x, \alpha)\right)$ and from (8) one gets

$$
\partial_{t} \tilde{J}_{l}+\left\{\omega_{l}, \tilde{J}_{l}\right\}_{x, k}=-2 \tilde{\gamma}_{l} \tilde{J}_{l}+\tilde{p}_{l},
$$

where $\{\cdot, \cdot\}_{x, k}$ denotes the $x, k$ Poisson Brackets and $\omega_{l}(t, x, k)$ is the eigenfrequency of the mode, while $\tilde{\gamma}_{l}$ and $\tilde{p}_{l}$ are the absorption coefficient and the emitted action density in canonical coordinates. This is the wave kinetic equation which usually is derived through the Wigner function formalism [1]. Both equation (8) and (9) describe the same transport process: indeed the transport of energy occurs along $\alpha$-parametrized surfaces (Lagrangian manifolds) given by $k=d_{x} \phi_{l}(t, x, \alpha)$ that are invariant under the Hamiltonian flow of the Hamiltonian function $\omega_{l}(t, x, k)$ according to Hamilton-Jacobi theory.

At last, let us consider the frequency $\omega=\omega_{l}(t, x, k)$ and the direction of the group velocity $s=v_{\mathrm{g}, l} /\left|v_{\mathrm{g}, l}\right|=s_{l}(t, x, k)$; in the high-frequency region of the spectrum the eigenfrequency $\omega_{l}$ is expected to be monotonous in $|k|$ while the direction of the group velocity $s$ is related to $k /|k|$; hence we make the hypothesis that $k=k(t, x, \omega, s)$, i.e., $(\omega, s)$ are in one-to-one correspondence to $k$ and they can be used as non-canonical momenta. Some algebra gives the specific intensity of radiation for the mode $l$, namely, $I_{l}(t, x, \omega, s)=\frac{n_{r, l}^{2} \omega^{3}}{(2 \pi)^{3} c^{2}} J_{l}(t, x, \alpha) / U_{l}(t, x, \alpha)$, where $n_{r, l}$ is the ray refractive index [4, 5], and from (8) one gets

$$
\frac{n_{r, l}^{2} \omega^{3}}{\left|v_{\mathrm{g}, l}\right|} \frac{\partial}{\partial t}\left(\frac{I_{l}}{n_{r, l}^{2} \omega_{l}^{3}}\right)+n_{r, l}^{2} \omega^{3} \frac{\partial}{\partial s}\left(\frac{I_{l}}{n_{r, l}^{2} \omega_{l}^{3}}\right)=j_{l}-\alpha_{l} I_{l},
$$

where $\alpha_{l}=2 \gamma_{l} /\left|v_{\mathrm{g}, l}\right|$ is the absorption coefficient and $j_{l}=n_{r, l}^{2} \omega^{3} P_{l} /\left((2 \pi)^{3} c^{2}\left|v_{\mathrm{g}, l}\right|\right)$ is the emission coefficient (the operators $\partial_{t}$ and $\partial_{s}$ act on both the implicit and explicit dependencies). This implies that the proper generalization of the Clausius adiabatic invariant for non-stationary plasmas is $C_{l}=I_{l} /\left(n_{r, l}^{2} \omega^{3}\right)$ in agreement with the conservation of the photon number per unit of phase-space volume [8].

\section{REFERENCES}

1. L. Ryzhik, G. Papanicolaou and J. B. Keller, Wave Motion 24, 327 (1996).

2. G. Bekefi, Radiation processes in plasmas, Wiley, N.Y.,1966.

3. L. A. Apresyan and Yu. A. Kravtsov, Radiation transfer, Gordon and Bresach Publishers, 1996.

4. T. H. Stix, Waves in plasmas, AIP, N.Y.,1992.

5. U. Bellotti, M. Bornatici and F. Engelmann, Rivista del Nuovo Cimento 201 (1997).

6. O. Maj and M. Bornatici, in Theory of Fusion Plasmas (SIF, Bologna 2002), pp.327-332.

7. R. G. Littlejohn and W. G. Flynn, Phys. Rev. A 44, 5239 (1991).

8. G. C. Pomraning, Equations of Radiation Hydrodynamics, Pergamon Press, Oxford, 1973. 